\title{
Filiform projections of the vestibular mucosa: What's your diagnosis?
}

\section{Mouna Ben Hamouda, Colandane Belajouza, Mohamed Denguezli}

\author{
Department of Dermatology, Farhat Hached University Hospital, Sousse-Tunisia
}

Corresponding author: Mouna Ben Hamouda, MD, E-mail: ben.hamouda.moona@gmail.com

\section{CASE REPORT}

29-year-old recently married woman was reffered by her gynecologist to our dermatology department for suspected vulvar condyloma acuminatum. She had no history of any extramarital sexual contacts and her partner was free of any clinical features of genital HPV infections. She was anxious about the origin of her lesions and the risk of her husband's contamination. She reported burning sensation, irritation and vulvar pain. On clinical examination soft digitate mucous colored papules with a smooth surface were noted at the vulvar vestibule. These papillomatous projections were pearly, uniformly arranged and had separate bases (Fig. 1). Dermatoscopy demonstrated regular and linear pinkish projections arranged in a symmetric distribution. Irregular linear vascular channels were observed (Fig. 2).

\section{WHAT'S YOUR DIAGNOSIS?}

\section{ANSWER: Vestibular Papillomatosis}

Vestibular papillomatosis (VP) is a normal variant of the female genital mucosa $[1,2]$. It is first described in 1981 by Altemeyer and named pseudocondylomata of the vulva [3]. VP is frequently misdiagnosed as condyloma acuminatum and presents a source of anxiety in patients, aggressive investigations and inappropriate treatment [2]. This entity is most often asymptomatic, but it can be accompanied, as our case, by a feeling of burning, pain or dyspareunia. To avoid the misdiagnosis of VP as genital warts, Moyal-Barranco et al. had proposed five clinical parameters [4]. Unlike condyloma acuminatum, VP is formed by regular, soft

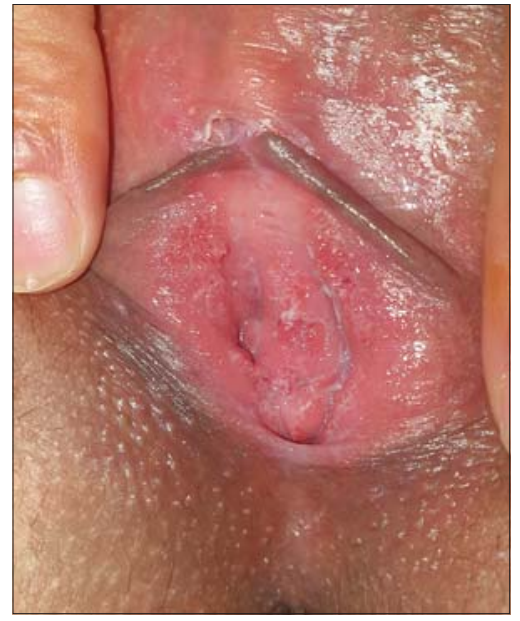

Figure 1: Clinical aspect: multiple skin-colored filiform andsoftprojections.

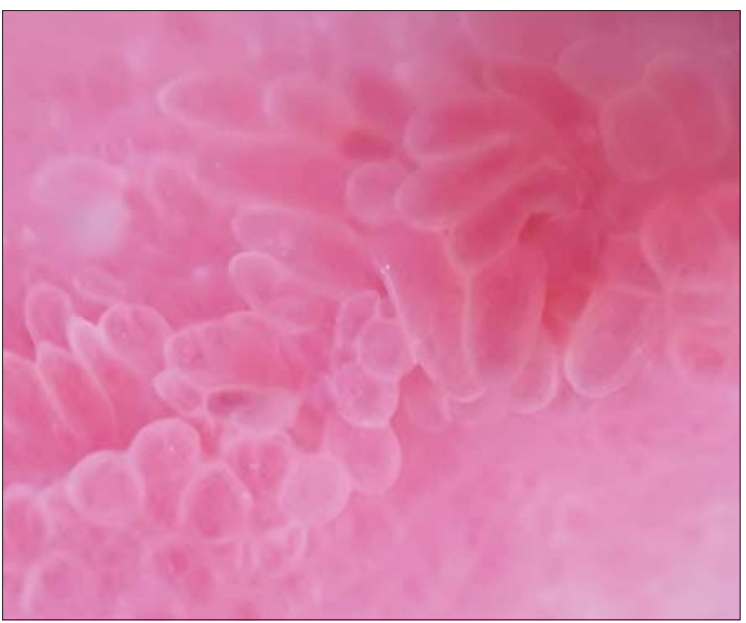

Figure 2: Dermatoscopy showing regular and linear pinkish projections arranged in a symmetric distribution.

and pink-colored papillae arising from a separate base. It is also characterized by the lack of circumscribed

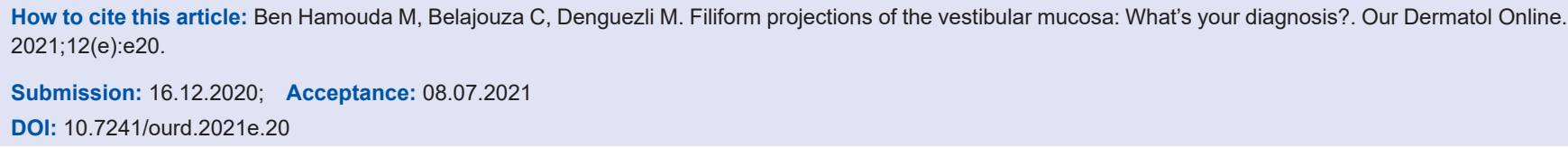


whitening on 5\% acetic acid application. Dermoscopy can help to differentiate these two conditions by allowing a better identification of these clinical parameters. Linear vessels may sometimes be observed in the transparent core of the finger-like projections [5]. On the other hand, dermoscopy of condyloma acuminatum shows irregular whitish projections with tapering end that arise from a common base and comprise conglomerate vessel. Based on these clinical criteria and the dermoscopic aspect, biopsies will be unnecessary to confirm the diagnosis of VP.

\section{Consent}

The examination of the patient was conducted according to the principles of the Declaration of Helsinki.

The authors certify that they have obtained all appropriate patient consent forms, in which the patients gave their consent for images and other clinical information to be included in the journal. The patients understand that their names and initials will not be published and due effort will be made to conceal their identity, but that anonymity cannot be guaranteed.

\section{REFERENCES}

1. Razmi T. Vestibular papillomatosis:a normal variation commonly misdiagnosed as genital condylomata. Am J Obstet Gynecol. 2019;220:403.

2. El Kadiri S, Bay Bay H, Chaoui R, Douhi Z, Elloudi S, Mernissi FZ. Two soulmates !!! Our Dermatol Online. 2020;11:e55.1-e55.2.

3. Altmeyer P. Chilf GN, Holzmann H. Hirsuties papiliaris vulvae (pseudocondylomata of the vulva). Hautarzt 1982;33:281-3.

4. Ozkur E, Falay T, Turgut Erdemir AV, Gurel MS, Leblebici C. Vestibular papillomatosis: An important differential diagnosis of vulvar papillomas. Dermatol Online J. 2016;22:13030/qt7933q377.

5. Giuffrida G, Lacarrubba F, Boscaglia S, Nasca MR, Micali G. A Noninvasive aid for office-based gynecologists for the diagnosis of common external genital disorders. Obstet Gynecol Int. 2019;16:1-5.

Copyright by Mouna Ben Hamouda, et al. This is an open access article distributed under the terms of the Creative Commons Attribution License, which permits unrestricted use, distribution, and reproduction in any medium, provided the original author and source are credited.

Source of Support: Nil, Conflict of Interest: None declared. 